\title{
Estimación de la radiación solar global, mediante temperaturas extremas, aplicando el modelo Bristow-Campbell en la región Junín, Perú
}

\author{
Estimation of global solar radiation, using extreme temperatures, \\ applying the Bristow-Campbell model in the Junín region

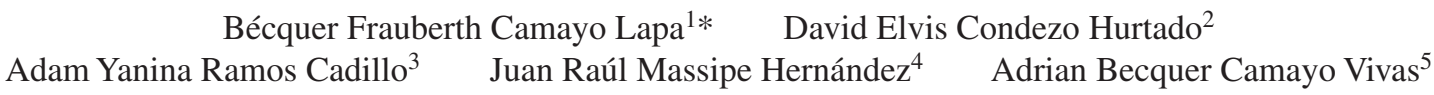 \\ Recibido 23 de febrero de 2018, aceptado 13 de septiembre de 2018 \\ Received: February 23, 2018 Accepted: September 13, 2018
}

\begin{abstract}
RESUMEN
Se planteó el objetivo principal de estimar la radiación solar global solar media diaria mensual y anual, mediante temperaturas extremas, aplicando el modelo Bristow-Campbell a las 19 estaciones meteorológicas de la Región Junín, responsabilidad del Servicio Nacional de Meteorología e Hidrología del Perú (SENAMHI) y los objetivos secundarios de calcular las temperaturas pronosticadas máximas y mínimas a través de series de tiempo. El método utilizado para la estimación de la radiación solar global media diaria mensual y anual fue el modelaje mediante el modelo empírico Bristow-Campbell y para el pronóstico de las temperaturas se utilizó la serie de tiempos mediante la técnica documental de los datos históricos de la página web de SENAMHI desde los años 2000 al 2015. Se determinó modelos predictivos estacionales para pronosticar temperaturas máximas y mínimas al 2017 de las 19 estaciones de la Región Junín. Con las temperaturas extremas pronosticadas se estimó la radiación solar global diaria media mensual mediante el modelo empírico Bristow-Campbell validado con datos registrados para estimar el coeficiente $\boldsymbol{a}$ considerando la altitud, consiguiendo que existen diferencias entre la Sierra y Selva siendo en la Sierra los valores promedios al $6 \mathrm{kWh} / \mathrm{m}^{2} /$ día y en la Selva $4 \mathrm{kWh} / \mathrm{m}^{2} /$ día, ambos valores resultan rentables para su aplicación con fines de producir calor, frío y electricidad con energía solar. Estos resultados validan el modelo Bristow-Campbell para estimar confiablemente la radiación solar global diaria media mensual y anual, mediante temperaturas extremas en la Región Junín - Perú.
\end{abstract}

Palabras clave: Radiación solar, modelo Bristow-Campbell, Radiación solar Junín Perú.

\section{ABSTRACT}

The main objective of the study was to estimate the solar mean global solar radiation monthly and annual, using extreme temperatures, applying the Bristow-Campbell model to the 19 meteorological stations of the Junin Region, under the responsibility of the National Service of Meteorology and Hydrology of Peru (SENAMHI) and the secondary objectives of calculating the maximum and minimum predicted temperatures through time series. The method used for the estimation of global mean daily solar radiation monthly and yearly was modeling using the empirical model Bristow-Campbell and for the forecast of

1 Facultad de Ciencias Aplicadas - UNCP. Aguirre Morales No 2096. Huancayo, Perú.

E-mail: camayobecquer@hotmail.com

2 Facultad de Ingeniería Eléctrica - UNCP. Huancayo, Perú. E-mail: davidelvis7@ hotmail.com

SENAMHI - JUNIN. Huancayo, Perú. E-mail: adalia04367@gmail.com

Alma Red Global. España. E-mail: raulm026@hotmail.com

5 Facultad de Ciencias Aplicadas - UNCP. CCHH Parra de Riego III Etapa Block 4 Dpto. 402. Huancayo, Perú.

E-mail: vivaadrian9@hotmail.com

* Autor de correspondencia: camayobecquer@hotmail.com 
temperatures was used the time series using the documentary technique of historical data of the website of SENAMHI from the years 2000 to 2015. It was determined seasonal predictive models to predict maximum and minimum temperatures to 2017 of the 19 stations of the Junin Region. With predicted extreme temperatures, global average daily solar radiation was estimated by means of the validated Bristow-Campbell empirical model with recorded data to estimate the coefficient a considering the altitude, obtaining that there are differences between the Sierra and Selva, being in the Sierra the average values at $6 \mathrm{kWh} / \mathrm{m}^{2} /$ day and in Jungle $4 \mathrm{kWh} / \mathrm{m}^{2} /$ day, both values are profitable for their application with the purpose of producing heat, cold and electricity with solar energy. These results validate the BristowCampbell model to reliably estimate monthly average and annual average global solar radiation, using extreme temperatures in the Junín Region - Peru.

Keywords: Solar radiation, model Bristow-Campbell, estimation solar radiation, solar radiation Junín.

\section{INTRODUCCIÓN}

El Perú es un lugar ideal para iniciar proyectos de energía renovable, debido a su gran abundancia de recursos y fuentes de energía verde: como el sol, el agua, la energía geotérmica, la eólica o la biomasa [1], para aportar beneficio a la sociedad, la fuente de energía posee, idealmente, las características de ser económicamente viable [2], como lo es el Sol como fuente de energía al alcance de todos. La radiación solar juega un papel importante en el cambio climático, el balance energético y las aplicaciones energéticas [3].

Estación Tarma, la National Aeronautics and Space Administration (NASA) reporta una radiación solar global promedio anual de $5,25 \mathrm{kWh} / \mathrm{m}^{2} /$ día y una altitud de 2635 m.s.n.m que difiere de los que reporta la estación Tarma, que se encuentra a 3034 m.s.n.m., que es de $5,67 \mathrm{kWh} / \mathrm{m}^{2} /$ día.

En la Región Junín, de las 19 estaciones meteorológicas a cargo del Servicio Nacional de Meteorología e Hidrología del Perú (SENAMHI), solo las estaciones de Santa Ana (provincia Huancayo), de Tarma (Provincia de Tarma) y de Satipo (Provincia de Satipo) cuentan con piranómetros, instrumentos que registran la radiación solar global. El mapa solar elaborado por SENAMHI 2003 [4] nos da informaciones de radiación solar global por departamentos y estaciones con mucho margen de error que no permiten tomar decisiones para aplicaciones puntuales [5].

Nuestro estudio sobre la estimación de la energía solar global es debido a que no se cuenta con datos confiables y puntuales en la Región Junín.
De los modelos empíricos de estimación de la radiación solar global citados por el Atlas de Energía Solar del Perú [4] se utilizó el modelo de BristowCampbell por adaptarse mejor a las condiciones y contar con valores de temperaturas extremas como lo corrobora el estudio de Camayo et al. [5].

Por otra parte, A. de la Casa. et al. [6] llega a la conclusión que la utilización de valores estimados de radiación solar global con el modelo (BristowCampbell) produce simulaciones similares a las que se obtiene con datos observados de radiación solar global; J. Alvarez et al. [7] estima la radiación solar global mensual utilizando los modelos BristowCampbell al igual que Torres et al [8] en el altiplano de central de Bolivia y Victor H. Quej et al. [9] recomienda que, si solo los datos de temperatura están disponibles para la estimación de la radiación solar global, el modelo de Bristow-Campbell obtiene los mejores resultados.

Wang [10] considera otros factores que influyen en la radiación solar incidente como la humedad relativa, la nubosidad, etc. en el modelo Bristow-Campbell. En la investigación presente no se ha considerado estos factores, lo que daría como resultado otra investigación para estudiar si es aplicable en nuestras condiciones de Junín.

Se planteó el objetivo principal de estimar la radiación solar global mediante temperaturas extremas, aplicando el modelo Bristow-Campbell a las 19 estaciones meteorológicas de la Región Junín.

Se consideró como objetivos secundarios: Elaborar una base de datos estimados de radiación solar global media diaria mensual y anual y de 
temperaturas máximas y mínimas a través de serie de tiempo.

Las 19 estaciones meteorológicas de SENAMHI Junín cuentan, en su página web, con datos de temperaturas máximas y mínimas desde el año 2000 hasta el año 2015, esto ha permitido pronosticar estas temperaturas para el 2017 a partir de las cuales se ha aplicado el modelo BristowCampbell validado con datos registrados para estimar el coeficiente $\boldsymbol{a}$ considerando la altitud, para estimar la radiación solar global diaria media mensual y anual.

\section{ANÁLISIS TEÓRICO}

\section{Radiación solar incidente}

Según [11], la caracterización de la radiación solar incidente sobre la superficie de la Tierra no es algo sencillo por tres razones: Por la aleatoriedad de la radiación solar, el movimiento relativo Sol-Tierra y la variedad de los modelos existentes.

La radiación solar global incidente sobre la superficie terrestre estaría viene dado por la expresión:

$$
R_{s}=R_{d i r}+R_{d i f}
$$

donde:

- $\quad R_{g}$ : radiación solar global, [W/m²];

- $\quad R_{d i r}$ : radiación solar directa, [W/m²];

- $R_{d i f}$ : radiación solar difusa, $\left[\mathrm{W} / \mathrm{m}^{2}\right]$.

En la Figura 1, se muestra los diferentes componentes que conforma la radiación solar total: directa, difusa y reflejada (albedo).

Esta relación determina la disponibilidad de la radiación solar global en una localidad específica. Cuando no se dispone de instrumentos de medición, debidamente calibrados, durante largos períodos de tiempo se recurre a métodos computacionales o empíricos, los cuales estiman el valor de la radiación solar global a partir de modelos estadísticos elaborados a partir de base de datos de variables climáticas [12].

\section{Métodos empíricos de estimación de la radiación solar}

Desde comienzos del siglo XX han existido esfuerzos por establecer modelos simples, que

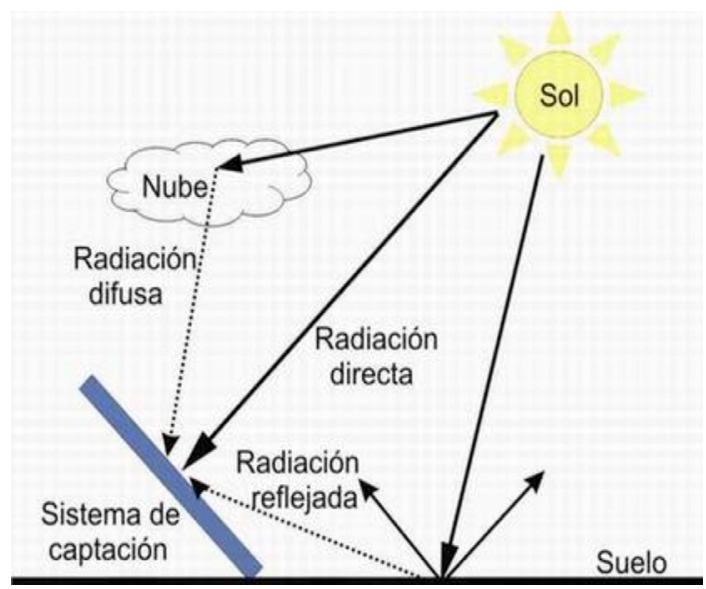

Figura 1. Tipos de radiación solar.

permitan estimar la radiación solar global sobre la superficie terrestre, a partir de la radiación solar extraterrestre y el estado de la atmósfera. Uno de los modelos más significativos es el de A.K. Angström 1924 [4], que estima la densidad de flujo diario de radiación global a partir de una ecuación de regresión lineal del tipo:

$$
\frac{H_{g}}{H_{o}}=a+b \cdot\left(\frac{n}{N}\right)
$$

- $H_{g}$ : radiación solar global, $\left[\mathrm{Wh} / \mathrm{m}^{2}\right]$;

- $H_{o}$ : radiación solar extraterrestre, $\left[\mathrm{Wh} / \mathrm{m}^{2}\right]$;

- $\quad n$ : horas de sol, [h];

- $\quad N$ : duración del día solar, [h].

Los coeficientes "a" y "b" están asociados al tipo de nubes y a la turbidez de la atmósfera. Angström sugirió los valores de 0,2 para el coeficiente "a" y de 0,5 para el coeficiente "b". A partir de esta descripción, Bristow y Campbell [4] desarrollaron un modelo (Ec. 3) para estimar la radiación solar global en función de la radiación solar extraterrestre y la diferencia de temperaturas extremas. Baigorria et al. (2003a) llegaron a la conclusión que el modelo Bristow-Campbell era el que mejor se adecuaba a las condiciones de Perú. Este modelo sugiere la estimación de la transmisividad o irradiación relativa $\left(H / H_{o}\right)$ como función de la diferencia entre las temperaturas máximas y mínimas:

$$
\frac{H_{g}}{H_{o}}=a \cdot\left(1-e^{-b \cdot\left(T_{\max }-T_{\min }\right)^{C}}\right)
$$


donde:

- $\mathrm{T}_{\text {máx }}$ : temperatura máxima promedio mensual, $\left[{ }^{\circ} \mathrm{C}\right]$;

- $\mathrm{T}_{\text {mín }}$ : temperatura mínima promedio mensual, $\left[{ }^{\circ} \mathrm{C}\right]$;

- a, b y c: coeficientes.

Los valores para cada coeficiente son de 0,7 para el coeficiente "a", entre 0,004 y 0,010 para el coeficiente "b" y 2,4 para el coeficiente "c" (Meza y Varas, 2000 citado por Atlas Energía Solar del Perú, 2003 [4]).

En este caso, los valores empíricos $(\mathbf{a}, \mathbf{b}$ y $\mathbf{c})$ tienen también un significado físico; a, representa el máximo valor característico de cada área y depende de la contaminación atmosférica y de la altitud; $\mathbf{b}\left({ }^{\circ} \mathrm{C}^{-1}\right)$ y c determinan el efecto del incremento de $\Delta T$ sobre la máxima " $\tau$ " de la atmósfera (Mesa y Varas, 2000 ; citado por citado por Atlas Energía Solar del Perú, 2003 [4]). En la Tabla 1 se muestra los resultados obtenidos con el programa de Excel del modelo B-C para estimar la radiación solar global de la Región de Junín.

\section{Modelo B - C en Excel para estimar la radiación solar global mensual (HS)}

Se ha desarrollado un programa en Excel del modelo Bristow-Campbell en Excel siguiendo el procedimiento propuesto por Camayo [4] cuyo algoritmo se muestra a continuación:

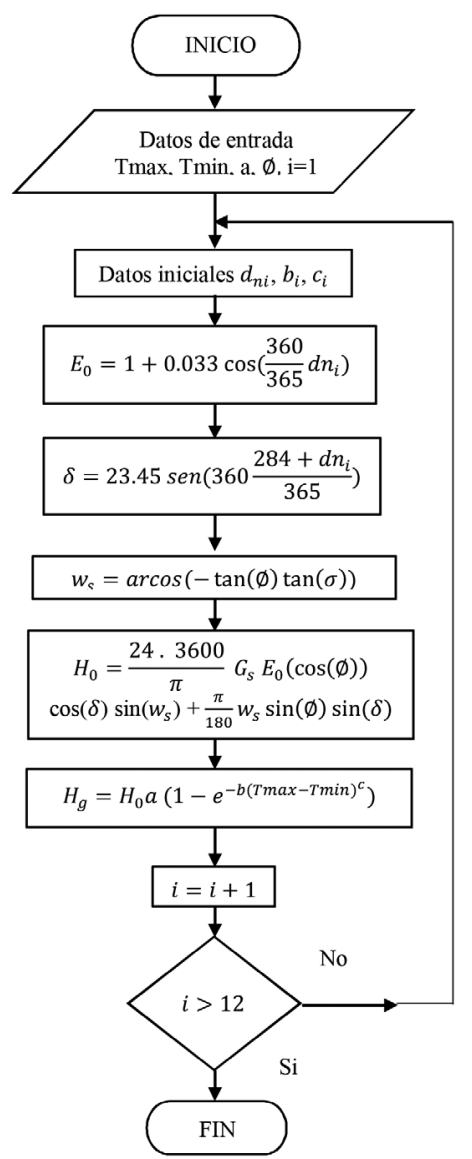

Tabla 1. Modelo Bristow-Campbell en Excel.

\begin{tabular}{|c|c|c|c|c|c|c|c|c|c|c|c|c|}
\hline \multirow[t]{2}{*}{ Región: } & \multicolumn{2}{|l|}{ JUNIN } & Altitud: & 2.872 & msnm & \multicolumn{3}{|c|}{ Latitud: $1^{\circ}{ }^{\circ} 9^{\prime}$ Sur } & \multicolumn{4}{|c|}{ a: 0,73} \\
\hline & \multicolumn{3}{|c|}{ Datos de Entrada } & \multicolumn{9}{|c|}{ Radiación Solar estimada } \\
\hline$d_{n}$ & $\mathbf{T}_{\max }$ & $\mathbf{T}_{\min }$ & Latitud & $\mathbf{E}_{0}$ & $\delta$ & $\omega \mathbf{S}_{\mathbf{h}}$ & Heh & $\mathbf{N}$ & cB & bB & Hsh & Hsh \\
\hline$[-]$ & {$\left[{ }^{\circ} \mathrm{C}\right]$} & {$\left[{ }^{\circ} \mathrm{C}\right]$} & [rad.] & {$[-]$} & [rad.] & [rad.] & $\begin{array}{c}{[\mathrm{MJ} / \mathrm{m} 2 /} \\
\text { día] }\end{array}$ & $\begin{array}{c}\text { [horas/ } \\
\text { día] }\end{array}$ & {$[-]$} & {$[-]$} & $\begin{array}{c}{[\mathrm{MJ} / \mathrm{m} 2 /} \\
\text { día] }\end{array}$ & $\begin{array}{c}{[\mathrm{kWh} / \mathrm{m} 2 /} \\
\text { día] }\end{array}$ \\
\hline 15 & 19,62 & 8,82 & $-0,207$ & 1,032 & $-0,370$ & 1,652 & 40,026 & 12,622 & 1,345 & 0,049 & 20,286 & 5,635 \\
\hline 46 & 19,26 & 9,08 & $-0,207$ & 1,023 & $-0,230$ & 1,620 & 39,518 & 12,375 & 1,384 & 0,045 & 19,445 & 5,401 \\
\hline 74 & 19,17 & 8,90 & $-0,207$ & 1,010 & $-0,047$ & 1,581 & 37,682 & 12,076 & 1,377 & 0,046 & 18,642 & 5,178 \\
\hline 105 & 19,88 & 7,70 & $-0,207$ & 0,992 & 0,166 & 1,536 & 34,041 & 11,732 & 1,240 & 0,061 & 18,355 & 5,098 \\
\hline 135 & 20,32 & 5,84 & $-0,207$ & 0,977 & 0,329 & 1,499 & 30,296 & 11,453 & 1,074 & 0,089 & 17,477 & 4,855 \\
\hline 166 & 20,01 & 4,41 & $-0,207$ & 0,968 & 0,407 & 1,480 & 28,209 & 11,309 & 0,993 & 0,109 & 16,703 & 4,640 \\
\hline 196 & 19,91 & 4,05 & $-0,207$ & 0,968 & 0,375 & 1,488 & 28,962 & 11,370 & 0,975 & 0,115 & 17,247 & 4,791 \\
\hline 227 & 20,41 & 4,75 & $-0,207$ & 0,976 & 0,239 & 1,520 & 32,140 & 11,610 & 0,989 & 0,110 & 19,057 & 5,294 \\
\hline 258 & 20,51 & 6,42 & $-0,207$ & 0,991 & 0,037 & 1,561 & 35,996 & 11,941 & 1,102 & 0,083 & 20,563 & 5,712 \\
\hline 288 & 20,76 & 7,64 & $-0,207$ & 1,008 & $-0,169$ & 1,607 & 38,630 & 12,273 & 1,172 & 0,070 & 21,481 & 5,967 \\
\hline 319 & 20,80 & 7,71 & $-0,207$ & 1,023 & $-0,335$ & 1,644 & 39,715 & 12,558 & 1,174 & 0,070 & 22,067 & 6,130 \\
\hline 349 & 19,82 & 8,53 & $-0,207$ & 1,032 & $-0,407$ & 1,661 & 39,942 & 12,691 & 1,304 & 0,053 & 20,785 & 5,774 \\
\hline Media & 20,04 & 6,99 & & & & & & & & & & 5,373 \\
\hline
\end{tabular}

Fuente: Modelo Bristow-Campbell en Excel validado por Camayo [5]. 
donde:

- $d n$ : día de referencia del mes año;

- $E_{0}$ : Corrección de la excentricidad de la órbita terrestre;

- $\quad \delta$ : declinación solar, [radianes];

- $\omega_{S}$ : ángulo horario de salida del Sol, [radianes];

- $G_{S C}$ : constante solar, $1367 \mathrm{~W} / \mathrm{m}^{2}$;

- $\varnothing$ : latitud, [radianes].

\section{MATERIALES Y MÉTODOS}

El método utilizado es el observacional de datos registrados meteorológicos como temperaturas máximas, mínimas diarias, promedios mensuales y anuales y datos registrados de radiación solar para identificar la relación de las características meteorológicas (temperaturas) con las actinométricas (radiación solar global) que puedan permitir el desarrollo de modelos de estimación de la radiación solar donde no existan informaciones actinométricas.

\section{Procedimiento para crear base datos de radiación solar global diaria, media mensual y anual:}

\section{Primera etapa:}

Se crea una base de datos de temperaturas extremas a partir de la página web de SENAMHI Junín desde el año 2000 hasta el año 2015 [13]. Estas temperaturas máximas y mínimas diarias son la base para pronosticar el año 2017, mediante series de tiempo.

\section{Segunda etapa:}

Se crea la base de datos de radiación solar global diaria y diaria media mensual, se utilizará el modelo BristowCampbell validado por Camayo [14] utilizando como insumo la base de datos de temperaturas extremas pronosticadas al 2017.

\section{Estaciones de estudio en la Región Junín}

En la Tabla 2, se muestra la latitud, longitud, altitud y valores de la constante "a" de las 19 estaciones meteorológicas de la Región Junín, datos que se utilizaron en la estimación de la radiación solar con el modelo empírico Bristow-Campbell.

\section{RESULTADOS}

\section{Resultados y análisis de temperaturas máximas y mínimas promedio mensual pronosticadas}

Modelos, precisión y gráficos de las estaciones Las temperaturas máximas y mínimas promedio mensual pronosticadas que resultaron con los datos históricos de 15 años desde el 2000 al 2015

Tabla 2. Datos de las 19 estaciones en la Región Junin.

\begin{tabular}{|l|c|c|c|c|l|}
\hline \multirow{1}{*}{ Estación } & Latitud & Longitud & Altitud & a & \multirow{2}{*}{ Clima } \\
\cline { 2 - 5 } & $(\mathbf{S})$ & $(\mathbf{O})$ & $\mathbf{m s n m}$ & {$[-]$} & \\
\hline Pichanaki & $10^{\circ} 56^{\prime}$ & $74^{\circ} 52^{\prime}$ & 514 & 0,560 & Semi húmedo y cálido \\
\hline Runatullo & $11^{\circ} 37^{\prime}$ & $75^{\circ} 00^{\prime}$ & 3498 & 0,780 & Moderadamente húmedo y semifrío \\
\hline Jauja & $11^{\circ} 47^{\prime}$ & $75^{\circ} 28^{\prime}$ & 3378 & 0,780 & Semi húmedo y semifrío \\
\hline Tarma & $11^{\circ} 23^{\prime}$ & $75^{\circ} 41^{\prime}$ & 3034 & 0,718 & Arido y semifrío \\
\hline Huasahuasi & $11^{\circ} 16^{\prime}$ & $75^{\circ} 38^{\prime}$ & 2765 & 0,718 & Seco y semifrío \\
\hline Comas & $11^{\circ} 44^{\prime}$ & $75^{\circ} 07^{\prime}$ & 3590 & 0,780 & Ligeramente húmedo y semifrío \\
\hline San Ramón & $11^{\circ} 07^{\prime}$ & $75^{\circ} 21^{\prime}$ & 888 & 0,600 & Ligeramente húmedo y cálido \\
\hline Sátipo & $11^{\circ} 13^{\prime}$ & $74^{\circ} 36^{\prime}$ & 590 & 0,558 & Ligeramente húmedo y cálido \\
\hline Puerto Ocopa & $11^{\circ} 08^{\prime}$ & $74^{\circ} 18^{\prime}$ & 336 & 0,600 & Seco y cálido \\
\hline La Oroya & $11^{\circ} 34^{\prime}$ & $75^{\circ} 57^{\prime}$ & 3957 & 0,780 & Semi húmedo y semifrío \\
\hline Ricran & $11^{\circ} 32^{\prime}$ & $75^{\circ} 31^{\prime}$ & 3580 & 0,780 & Semi húmedo y semifrío \\
\hline Ingenio & $11^{\circ} 52^{\prime}$ & $75^{\circ} 16^{\prime}$ & 3450 & 0,780 & Semi húmedo y semifrío \\
\hline Junín & $11^{\circ} 08^{\prime}$ & $75^{\circ} 59^{\prime}$ & 4114 & 0,820 & Muy húmedo y semifrío \\
\hline San Juan de Jarpa & $12^{\circ} 07^{\prime}$ & $75^{\circ} 25^{\prime}$ & 3671 & 0,800 & Ligeramente húmedo y semifrío \\
\hline Viques & $12^{\circ} 09^{\prime}$ & $75^{\circ} 13^{\prime}$ & 3218 & 0,800 & Seco y semifrío \\
\hline Huayao & $12^{\circ} 02^{\prime}$ & $75^{\circ} 19^{\prime}$ & 3328 & 0,780 & Semi húmedo y semifrío \\
\hline Laive & $12^{\circ} 15^{\prime}$ & $75^{\circ} 21^{\prime}$ & 3842 & 0,780 & Moderadamente húmedo y semifrío \\
\hline Santa Ana & $12^{\circ} 00^{\prime}$ & $75^{\circ} 13^{\prime}$ & 3298 & 0,671 & Semi húmedo y semifrío \\
\hline Shullcas & $10^{\circ} 02^{\prime}$ & $75^{\circ} 17^{\prime}$ & 3510 & 0,780 & Semi húmedo y semifrío \\
\hline & & & 2872 & 0,730 & \\
\hline
\end{tabular}


al aplicar serie de tiempos con el programa SPSS 22, se encontraron los siguientes:

a) Descripción del modelo: se encontró un modelo estacional simple sin ninguna tendencia para todas las estaciones.

b) Error medio cuadrático (RMSE)

RMSE $=0,6$ a 0,9 .

Información del error medio cuadrático estadísticamente significativo con error menores de 0,05 .

c) Parámetros del modelo.

$X_{t}=\alpha+E_{t} * R_{t}$

d) Gráfica del valor real y el valor pronosticado.

En la Figura 2, se muestra las temperaturas históricas máximas y mínimas del 2000 hasta el 2016 y las temperaturas pronosticadas del 2017 de la estación meteorológica de la estación Huayao.

\section{Resumen de los resultados de las temperaturas máximas y mínimas pronosticadas}

Se observa, que las temperaturas máximas y mínimas disminuyen en los meses de mayo, junio y julio considerados como invierno con respecto a la diferencia entre la temperatura máxima y mínima en la Sierra llegando como máxima promedio anual a $21,0^{\circ} \mathrm{C}$ en la estación Santa Ana y la temperatura mínima promedio anual de $0,1{ }^{\circ} \mathrm{C}$ es de la estación Junín. La diferencia entre la temperatura máxima y mínima promedio anual en la Selva es de $31,8^{\circ} \mathrm{C}$ que es en la estación Satipo y temperatura mínima de $18,0^{\circ} \mathrm{C}$ que es en la estación de San Ramón. Estas temperaturas extremas sirvieron como insumo para introducir al modelo Bristow-Campbell y estimar la radiación solar diaria media mensual por estaciones.

\section{Resultados y análisis de la radiación solar global estimada de las estaciones en estudio.}

El final del trabajo fue llegar a contar una base de datos radiación solar global diaria media mensual y anual estimada de las estaciones en estudio que se presenta en la Tabla 3. Se observa que el promedio de radiación solar global diaria media anual en la Región Junín es de $5,35 \mathrm{kWh} / \mathrm{m}^{2} /$ día, destacando la estación Junín con $6,53 \mathrm{kWh} / \mathrm{m}^{2} /$ día que pertenece a la sub región Sierra. En Selva Alta, la estación San Ramón llego a 4,50 kWh/m²/día y en la Selva Baja el promedio fue de $4,10 \mathrm{kWh} / \mathrm{m}^{2} /$ día, En las estaciones de la Sierra y la Selva disminuye un poco la radiación solar global en invierno que son los meses de mayo a julio y se incrementa en los demás meses.

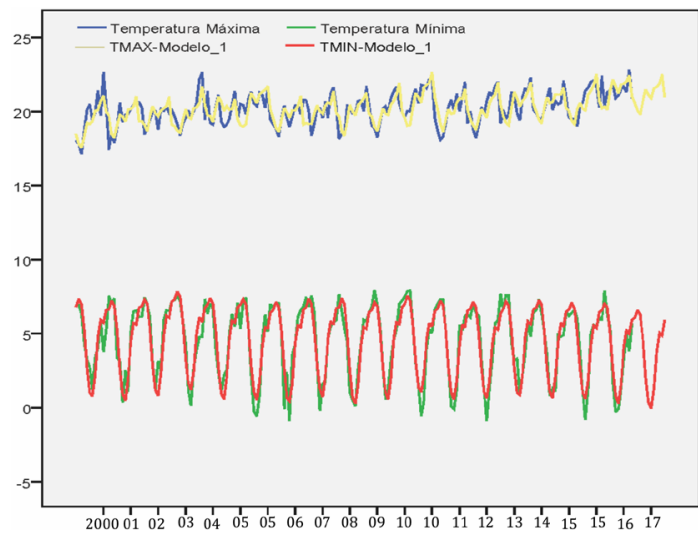

Figura 2. Temperaturas históricas máximas y mínimas de la estación Huayao.

\section{DISCUSIÓN}

Cuando hay necesidad de dimensionado de sistemas solares, hasta el momento, se recurre a los datos de la web de la National Aeronautics and Space Administration (NASA); donde reportan datos meteorológicos y de radiación solar a partir de la latitud y longitud geográficas. Al comparar, como ejemplo, con la estación Tarma, la NASA reporta una radiación solar global promedio anual de 5,25 $\mathrm{kWh} / \mathrm{m}^{2} /$ día y una altitud de 2635 m.s.n.m que difiere de los que reporta la estación Tarma, que se encuentra a 3034 m.s.n.m., que es de 5,67 kWh/ $\mathrm{m}^{2} /$ día, lo que nos motivó a desarrollar y validar el modelo empírico Bristow-Campbell para nuestra región de Junín.

El modelo Bristow-Campbell presenta mejor capacidad predicativa a escala de estimación de la radiación solar diaria media mensual cuando solo se dispone de temperaturas máximas y mínimas diarias, como se muestra en el estudio realizado. En el Atlas de Energía Solar del Perú se utilizó el modelo de Ångström-Prescott con información diaria de las estaciones con instrumentación piranométrica y actinométrica, por eso solo evaluaron la Estación de Cosmos y la de Huayao de la Región Junín y por estaciones climáticas con aproximaciones a $\pm 500 \mathrm{Wh} / \mathrm{m}^{2} /$ día que resulta poco confiable para aplicaciones de generación eléctrica.

En el contexto de los modelos de estimación de la radiación solar de naturaleza menos compleja, los que emplean como variable independiente es 
Tabla 3. Radiación solar diaria media mensual estimada mediante el modelo Bristow-Campbell de las estaciones con temperaturas máximas y mínimas pronosticadas del 2017 en la Región Junín, [kWh/m²/día].

\begin{tabular}{|c|c|c|c|c|c|c|c|c|c|c|c|c|c|c|}
\hline $\mathbf{N}^{\circ}$ & Estación & Ene & Feb & Mar & Abr & May & Jun & Jul & Ago & Sep & Oct & Nov & Dec & Prom. \\
\hline 1 & Pichanaki & 4,15 & 3,99 & 3,99 & 3,73 & 3,41 & 3,31 & 3,47 & 3,93 & 4,38 & 4,46 & 4,37 & 4,12 & 3,94 \\
\hline 2 & Runatullo & 4,39 & 3,93 & 4,21 & 4,09 & 4,24 & 4,12 & 4,28 & 4,79 & 4,84 & 4,56 & 4,68 & 4,26 & 4,36 \\
\hline 3 & Jauja & 6,09 & 5,83 & 5,50 & 5,55 & 5,41 & 5,22 & 5,39 & 5,89 & 6,23 & 6,45 & 6,59 & 6,15 & 5,86 \\
\hline 4 & Tarma & 6,23 & 6,12 & 5,89 & 5,48 & 4,98 & 4,72 & 4,87 & 5,36 & 5,81 & 6,12 & 6,29 & 6,15 & 5,67 \\
\hline 5 & Huasahuasi & 4,70 & 4,22 & 4,30 & 4,46 & 4,31 & 4,18 & 4,26 & 4,72 & 4,98 & 5,05 & 5,22 & 4,72 & 4,59 \\
\hline 6 & Comas & 6,32 & 6,01 & 5,77 & 5,53 & 5,17 & 4,90 & 5,05 & 5,63 & 6,10 & 6,37 & 6,64 & 6,39 & 5,82 \\
\hline 7 & San Ramón & 4,75 & 4,64 & 4,54 & 4,27 & 3,92 & 3,67 & 3,88 & 4,35 & 4,89 & 5,23 & 5,08 & 4,78 & 4,50 \\
\hline 8 & Sátipo & 4,32 & 4,23 & 4,19 & 3,93 & 3,48 & 3,38 & 3,57 & 4,02 & 4,45 & 4,63 & 4,60 & 4,39 & 4,10 \\
\hline 9 & Puerto Ocopa & 4,17 & 4,02 & 4,01 & 3,85 & 3,50 & 3,39 & 3,62 & 4,18 & 4,63 & 4,74 & 4,66 & 4,29 & 4,09 \\
\hline 10 & La Oroya & 6,21 & 5,88 & 5,46 & 5,53 & 5,37 & 5,15 & 5,33 & 5,80 & 6,16 & 6,45 & 6,70 & 6,28 & 5,86 \\
\hline 11 & Ricran & 5,27 & 4,87 & 4,47 & 4,56 & 4,80 & 4,72 & 4,89 & 5,28 & 5,52 & 5,64 & 5,70 & 5,02 & 5,06 \\
\hline 12 & Ingenio & 6,49 & 6,13 & 5,80 & 5,64 & 5,41 & 5,18 & 5,39 & 5,93 & 6,28 & 6,59 & 6,77 & 6,46 & 6,01 \\
\hline 13 & Junín & 6,94 & 6,58 & 6,36 & 6,09 & 5,76 & 5,62 & 5,94 & 6,59 & 6,93 & 7,09 & 7,42 & 7,00 & 6,53 \\
\hline 14 & San Juan de Jarpa & 5,99 & 5,49 & 5,15 & 5,28 & 5,26 & 5,02 & 5,17 & 5,73 & 6,06 & 6,30 & 6,61 & 6,02 & 5,67 \\
\hline 15 & Viques & 6,57 & 6,25 & 6,06 & 5,94 & 5,61 & 5,37 & 5,53 & 6,05 & 6,61 & 6,76 & 6,97 & 6,58 & 6,19 \\
\hline 16 & Huayao & 6,87 & 6,60 & 6,30 & 6,05 & 5,80 & 5,58 & 5,76 & 6,28 & 6,65 & 6,98 & 7,32 & 6,95 & 6,43 \\
\hline 17 & Laive & 6,42 & 6,09 & 5,81 & 5,74 & 5,52 & 5,51 & 5,72 & 6,24 & 6,43 & 6,65 & 6,99 & 6,49 & 6,13 \\
\hline 18 & Santa Ana & 5,89 & 5,79 & 5,45 & 5,29 & 5,05 & 4,88 & 5,07 & 5,50 & 5,78 & 6,00 & 6,26 & 5,94 & 5,58 \\
\hline 19 & Shullcas & 5,22 & 4,62 & 4,41 & 5,19 & 4,70 & 4,74 & 4,95 & 5,39 & 5,64 & 5,75 & 5,76 & 5,54 & 5,16 \\
\hline & & 5,63 & 5,33 & 5,14 & 5,06 & 4,83 & 4,67 & 4,85 & 5,35 & 5,70 & 5,89 & 6,03 & 5,66 & 5,35 \\
\hline
\end{tabular}

la heliofonia relativa o la nubosidad, presentan mejor capacidad predicativa a escala diaria que los procedimientos basados en la amplitud térmica, por lo que modelo Angström-Prescott es poco práctico para la mayoría de las estaciones meteorológicas por no con contar datos de heliofonia relativa.

Con respecto a las temperaturas medias y extremas, se encuentran registrados en SENAMHI en forma de manual, los publicados en su página web [13] no están actualizados, solo cuentan desde los años de 2000 hasta el 2015 por ello se ha tenido que, a través de series de tiempo, crear modelos predictivos para pronosticar las temperaturas máximas y mínimas del año 2017, pudiéndose considerar permanente esta información de temperaturas extremas ya que los modelos son estacionales sin tendencias por lo que se necesita estudios de temperaturas de históricas mayores de 40 años para estimar la variabilidad climática.

La Tabla 4, nos muestra que las radiaciones solares global pronosticadas al 2010 son homogéneas al comparar con las radiaciones globales al 2017 con un p-valor 0,602 y están relacionados con un coeficiente de correlación de Pearson de 0,93, lo que nos confirman la validez de las radiaciones solares globales estimadas para la Región Junín.

Con respecto a las radiaciones solares globales estimados en la región Junín son significativamente confiables al $95 \%$.

Se encontró diferencias significativas entre los valores promedios de radiación solar de la Sierra y la Selva. En la Sierra se alcanzo valores del orden de los $6 \mathrm{kWh} / \mathrm{m}^{2} /$ día, y en la Selva de $4 \mathrm{kWh} / \mathrm{m}^{2} /$ día. Consideramos que ambos valores son rentables para aplicaciones térmicas y de generación de electricidad con energía solar.

La información generada en esta investigación servirá para concientizar a las autoridades regionales, locales y sectoriales de considerar dentro de los planes y programas, el uso doméstico e industrial de la energía solar como fuente de energía y cumplir las metas del plan estratégico nacional al 2021 de considerar un incremento del $5 \%$ anual con energías renovables en la matriz energética nacional [15]. Además, a las instituciones públicas y privadas que 
Tabla 4. Comparación radiación solar global promedio anual 2010 [14] y 2017 pronosticados.

\begin{tabular}{|l|c|c|}
\hline \multirow{2}{*}{\multicolumn{1}{|c|}{ Estación }} & \multicolumn{2}{c|}{$\begin{array}{c}\text { Radiación solar, } \\
\text { [kWh/m2/día] }\end{array}$} \\
\cline { 2 - 3 } & $\mathbf{2 0 1 0}$ & $\mathbf{2 0 1 7}$ \\
\hline Pichanaki & 3,83 & 3,94 \\
\hline Runatullo & 4,60 & 4,36 \\
\hline Jauja & 5,98 & 5,86 \\
\hline Tarma & 5,61 & 5,67 \\
\hline Huasahuasi & 4,51 & 4,59 \\
\hline Comas & 4,96 & 5,82 \\
\hline San Ramón & 4,69 & 4,50 \\
\hline Sátipo & 3,98 & 4,10 \\
\hline Puerto Ocopa & 4,32 & 4,09 \\
\hline La Oroya & 5,92 & 5,86 \\
\hline Ricran & 4,96 & 5,06 \\
\hline Ingenio & 6,08 & 6,01 \\
\hline Junín & 5,96 & 6,53 \\
\hline San Juan de Jarpa & 5,90 & 5,67 \\
\hline Viques & 6,23 & 6,19 \\
\hline Huayao & 6,13 & 6,43 \\
\hline Laive & 6,09 & 6,13 \\
\hline Santa Ana & 5,37 & 5,58 \\
\hline Shullcas & 5,72 & 5,16 \\
\hline & $\mathbf{5 , 3 1}$ & $\mathbf{5 , 3 5}$ \\
\hline
\end{tabular}

promueven y aplican tecnologías con energía solar les servirá como fuente de información confiable para sus proyectos.

\section{CONCLUSIONES}

El modelo Bristow-Campbell permitió estimar la radiación solar global diaria media mensual y anual, mediante temperaturas extremas de las 19 estaciones meteorológicas de responsabilidad del SENAMHI del Perú (SENAMHI) región Junín 2017.

Las temperaturas pronosticadas de máximas y mínimas a través de serie de tiempo fueron con datos de 15 años desde el año 2000 hasta el 2015 de las 19 Estaciones de la Región Junín.

La radiación global solar media diaria mensual y anual mediante el modelo Bristow-Campbell se estimó con temperaturas promedio mensuales pronosticadas al 2017 considerando la altitud al estimar coeficiente $\boldsymbol{a}$ de las 19 Estaciones de la Región Junín siendo mayores en la Sierra con promedios de $6 \mathrm{kWh} / \mathrm{m}^{2} /$ día que en la selva con un promedio de $4 \mathrm{kWh} / \mathrm{m}^{2} /$ día.

La cuantificación de la disponibilidad de la energía solar sirve de base para que sea posible el diseño de políticas y medidas para incentivar el mayor uso de estas energías limpias que promuevan el desarrollo sostenible de la Región Junín y de todo Perú.

\section{REFERENCIAS}

[1] Pedro Gamio Ayta,. "Energías renovables y cambio climático". Konrad Adenauer Stiftung. Lima, Perú. 2015.

[2] Jordi Morató, Hermilio Salas, Nicola Salas y Jorge Montoya. "Reciclar Ciudad: Repensar la transformación de las ciudades". Catedra UNESCO. Universitat Politécnica de Catalunya. Ed. 2016. Barcelona. España.

[3] Ling Zou, Lunche Wang, Li Xia, Aiwen Lin, Bo Hu y Hongji Zhu. "Prediction and comparison of solar radiation using improved empirical models and Adaptive Neuro-Fuzzy Inference Systems". Renewable Energy. Volumen 106, pp. 343-353. June 2017.

[4] Servicio Nacional de Meteorología e Hidrología (SENAMHI). Ministerio de Energía y Minas. Atlas de Energía Solar en el Perú. Lima; 2003.

[5] Becquer Camayo Lapa, et al. Desarrollo del modelo Bristow-Campbell para estimar la radiación solar global de la región de Junín, Perú. $R T Q$ [online]. Vol. $35 \mathrm{~N}^{\circ}$ 2, pp. 220234. [citado 2017-10-27]. 2015.

[6] A. De La Casa, A. Rodríguez, G. Ovando. "Estimación de la radiación solar global en la provincia de Córdoba, Argentina, y su empleo en un modelo de rendimiento potencial de papa”. RIA. Revista de Investigaciones Agropecuaria.

[7] J. Alvarez, H. Mitasova and L. Allen. "Estimating Monthly Solar Radiation in South-Central Chile". Chilean J. Agric. Res. [online]. 2011, vol. $71 \mathrm{~N}^{\circ} 4$ [citado 201611-26], pp. 601-609.

[8] R. Torres, A. Burgoa y E. Ricaldi. "Modelos de estimación de la radiación solar para el Altiplano Central de Bolivia”. Revista Boliviana de Física [online]. Vol. 23 N²3, pp. 1-7. 2013.

[9] Victor H. Quej, Javier Almorox, Mirzakhayot Ibrakhimov and Laurel Saito. "Empirical 
models for estimating daily global solar radiation in Yucatán Peninsula, Mexico". Energy Conversion and Management 110 (2016) 448-456.

[10] Lunche Wang, Ozgur Kisi, Mohammad Zounemat-Kermani, Germán Ariel Salazar, Zhongmin Zhu and Wei Gong. "Solar radiation prediction using different techniques: model evaluation and comparison". Renewable and Sustainable Energy Reviews. Vol. 61, p. 384-397. August 2016.

[11] M. Castro y A. Colmenar. "Energía Solar Térmica y de Baja Temperatura". $3^{\text {a }}$ ed. España; Progensa; 2008.
[12] L. Jutglar. "Energía solar: Energías Alternativas y Medio Ambiente". España; Ceac; 2014.

[13] SENAMHI. http://www.senamhi.gob.pe/ main_mapa.php?t=dHi

[14] B. Camayo. "Radiación solar y calidad ambiental de la región de Junín". Universidad Nacional del centro del Perú. Huancayo, Perú, 2013.

[15] Centro Nacional de Planeamiento estratégico (CEPLAN). Plan Bicentenario: El Perú hacia el 2021. Lima, Perú 2011. 282 p. Ubicado en: https://www.mef.gob.pe/ contenidos/acerc_mins/doc_gestion/ PlanBicentenarioversionfinal.pdf 